\title{
A joint crisis plan negotiated with mental health staff significantly reduces compulsory admission and treatment in people with severe mental illness
}

Henderson C, Flood C, Leese $M$, et al. Effect of joint crisis plans on use of compulsory treatment in psychiatry: single blind randomised controlled trial. BMJ 2004;329:136.

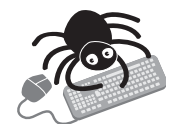

This article

contains extra text on the EBMH website

Does a joint crisis plan reduce the use of inpatient services, and compulsory admission and treatment in people with severe mental illness?

\section{METHODS}

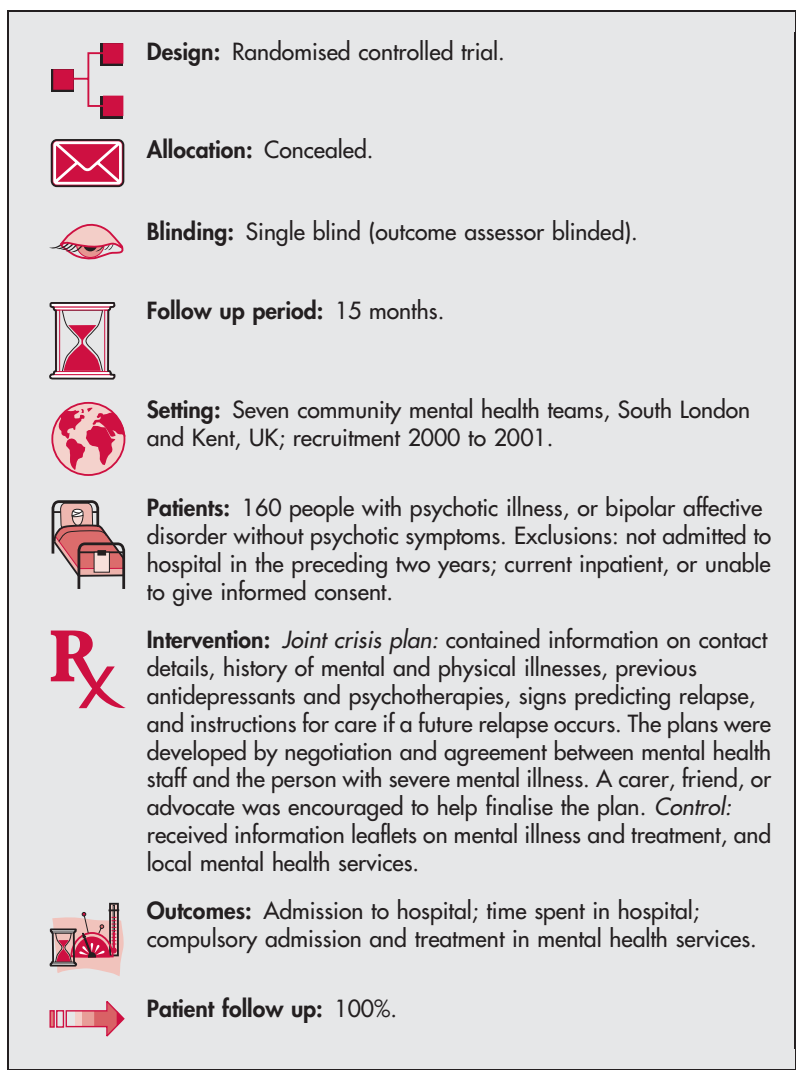

\section{MAIN RESULTS}

At 15 months, people with a joint crisis plan were significantly less likely to experience compulsory admission and treatment ( $13 \%$ v $27 \%$, RR $0.48,95 \% \mathrm{Cl} 0.24$ to 0.95 ) and there was a trend towards fewer overall admissions compared with control ( $30 \% v 44 \%$, RR $0.69,95 \% \mathrm{Cl}$ 0.45 to 1.04). The mean length of hospital stay after compulsory admission was significantly less for people with a joint crisis plan than controls (see http://www.ebmentalhealth.com/supplemental for table). There was no significant difference in the time spent in hospital for each group after non-compulsory hospital admission. The reasons for compulsory admission were similar for both groups, but people with a joint treatment plan were more likely to be released from hospital early after compulsory admission than controls.

For correspondence: Claire Henderson, Health Services Research Department, Institute of Psychiatry, King's College London, UK; hendersc@nypdrat.cpmc.columbia.edu

Sources of funding: Medical Research Council Training Fellowship in Health Service Research and South London and Maudsley Trust Health Service Research Committee, UK.

\section{CONCLUSIONS}

A joint crisis plan made with the help of mental health staff and a carer, friend, or advocate significantly reduces compulsory hospital admission and treatment in people with severe mental illness.

\section{Commentary}

nvoluntary admissions in England have increased by $57 \%$ over

10 years. ${ }^{1}$ Compulsory community treatment has had most attention as an alternative. This article examines another: joint crisis plans developed by patients and their mental health team, including an advance agreement for treatment in an emergency when patients are too unwell to give consent.

Henderson et al showed that this intervention significantly reduced compulsory admissions in patients with severe mental illness. The reduction in overall admission only just fell short of statistical significance $(p=0.07)$. However, the study was small and power was further reduced by the lower than anticipated admission rate, a key outcome. There are caveats. Barely one third participated. Although there were no significant differences in age, sex, and length of contact between participants and non-participants, there were no data on ethnicity of non-participants. This is important given the high rates of involuntary admission in people of African-Caribbean descent, and their overrepresentation in secure units and special hospitals. ${ }^{2}{ }^{3}$ The nature of the intervention meant that it was only single-blind, and allocation concealment in the randomisation was unclear.

Against this, the sample came from a range of settings (inner city, suburban, small town) and participants showed broad ethnic representation.

These findings are different to those from another similar sized study in the London area where advance directives did not reduce involuntary admission. ${ }^{4}$ One explanation might be that the study of Henderson et al had a lower participation rate leading to selection bias: patients with a better prognosis may have differentially participated. Another is the intensity of their intervention: the plan was developed at a 30-50 minute meeting with the researcher, treating team, patient, and invited relative. In the other study, patients were only given booklets with seven statements for completion. ${ }^{4}$ This study needs replication but offers better proof than the evidence for compulsory community treatment. ${ }^{5}$ Steve Kisely MD, MSc, FRANZCP, FRCPC, FAFPHM Chair in Health Outcomes, Departments of Psychiatry, Community Health \& Epidemiology, Dalhousie University, Canada

1 Department of Health. Inpatients formally detained in hospitals under the Mental Health Act 1983 and other legislation, England: 1988-89 to 1998-99. London: Government Statistical Service, 1999.

2 McColl AJ. Schizophrenia. In: McColl AJ, Gulliford MC, eds. Population Health Outcome Indicators for the NHS: a feasibility study. London: Faculty of Public Health Medicine, 1993.

3 Koffman J, Fulop NJ, Pashley D, et al. Ethnicity and use of acute psychiatric beds: one-day survey in North and South Thames regions. Br J Psychiatry 1997; 171:238-41.

4 Papageorgiou A, King M, Janmohamed A, et al. Advance directives for patients compulsorily admitted to hospital with serious mental illness.

Randomised controlled trial. Br J Psychiatry 2002;181:513-19.

5 Bindman J. Involuntary outpatient treatment in England and Wales. Curr Opin Psychiatry 2002;15:595-8. 